\title{
Article \\ Effects of Transcranial Ultrasound Stimulation on Trigeminal Blink Reflex Excitability
}

\author{
Andrea Guerra ${ }^{1}$, Edoardo Vicenzini ${ }^{2}$, Ettore Cioffi ${ }^{2}$, Donato Colella ${ }^{2}$, Antonio Cannavacciuolo ${ }^{2}$, Silvia Pozzi ${ }^{3}$, \\ Barbara Caccia $^{3}$, Giulia Paparella ${ }^{1}$, Giulia Di Stefano ${ }^{2}$, Alfredo Berardelli ${ }^{1,2}$ and Matteo Bologna ${ }^{1,2, *}$ \\ 1 IRCCS Neuromed, 86077 Pozzilli, Italy; andrea.guerra@uniroma1.it (A.G.); \\ giulia.paparella@uniroma1.it (G.P.); alfredo.berardelli@uniroma1.it (A.B.) \\ 2 Department of Human Neurosciences, Sapienza University of Rome, 00185 Rome, Italy; \\ edoardo.vicenzini@uniroma1.it (E.V.); ettore.cioffi@uniroma1.it (E.C.); donato.colella@uniroma1.it (D.C.); \\ antonio.cannavacciuolo@uniroma1.it (A.C.); giulia.distefano@uniroma1.it (G.D.S.) \\ 3 National Center for Radiation Protection and Computational Physics, Istituto Superiore di Sanità (ISS), \\ 00161 Rome, Italy; silvia.pozzi@iss.it (S.P.); barbara.caccia@iss.it (B.C.) \\ * Correspondence: matteo.bologna@uniroma1.it; Tel.: +39-06-49914074
}

check for updates

Citation: Guerra, A.; Vicenzini, E.; Cioffi, E.; Colella, D.; Cannavacciuolo, A.; Pozzi, S.; Caccia, B.; Paparella, G.; Di Stefano, G.; Berardelli, A.; et al. Effects of Transcranial Ultrasound Stimulation on Trigeminal Blink Reflex Excitability. Brain Sci. 2021, 11, 645. https://doi.org/10.3390/ brainsci11050645

Academic Editor: François Tremblay

Received: 2 April 2021

Accepted: 12 May 2021

Published: 15 May 2021

Publisher's Note: MDPI stays neutral with regard to jurisdictional claims in published maps and institutional affiliations.

Copyright: (C) 2021 by the authors Licensee MDPI, Basel, Switzerland. This article is an open access article distributed under the terms and conditions of the Creative Commons Attribution (CC BY) license (https:/ / creativecommons.org/licenses/by/ $4.0 /)$.

\begin{abstract}
Recent evidence indicates that transcranial ultrasound stimulation (TUS) modulates sensorimotor cortex excitability. However, no study has assessed possible TUS effects on the excitability of deeper brain areas, such as the brainstem. In this study, we investigated whether TUS delivered on the substantia nigra, superior colliculus, and nucleus raphe magnus modulates the excitability of trigeminal blink reflex, a reliable neurophysiological technique to assess brainstem functions in humans. The recovery cycle of the trigeminal blink reflex (interstimulus intervals of 250 and $500 \mathrm{~ms}$ ) was tested before (T0), and 3 (T1) and $30 \mathrm{~min}$ (T2) after TUS. The effects of substantia nigra-TUS, superior colliculus-TUS, nucleus raphe magnus-TUS and sham-TUS were assessed in separate and randomized sessions. In the superior colliculus-TUS session, the conditioned R2 area increased at $\mathrm{T} 1$ compared with $\mathrm{T} 0$, while $\mathrm{T} 2$ and $\mathrm{T} 0$ values did not differ. Results were independent of the interstimulus intervals tested and were not related to trigeminal blink reflex baseline (T0) excitability. Conversely, the conditioned R2 area was comparable at T0, T1, and T2 in the nucleus raphe magnusTUS and substantia nigra-TUS sessions. Our findings demonstrate that the excitability of brainstem circuits, as evaluated by testing the recovery cycle of the trigeminal blink reflex, can be increased by TUS. This result may reflect the modulation of inhibitory interneurons within the superior colliculus.
\end{abstract}

Keywords: transcranial ultrasound stimulation (TUS); blink reflex; brainstem; excitability; GABA; interneurons; non-invasive brain stimulation; neuromodulation

\section{Introduction}

Transcranial ultrasound stimulation (TUS) is a novel non-invasive brain stimulation technique that uses acoustic pressure waves delivered deep within tissues [1-3]. Ultrasound waves can be focused on a particular target with a specific spatial resolution based on wavelength and frequency. TUS can be transmitted through the skull and may exert direct modulation of neurons at low intensities [2-4]. The putative mechanism of TUS relates to the mechanical energy of ultrasound, which produces changes in cell membrane proteins and bioelectrical properties, including activation of voltage-dependent sodium and potassium, and calcium channels [5-7].

In humans, TUS of the primary motor cortex decreased corticospinal excitability and intracortical facilitation, increased GABAergic inhibition and reduced motor reaction task performance [8,9]. Conversely, motor evoked potential (MEP) amplitude was facilitated immediately after stimulation [10]. When delivered over the primary somatosensory cortex, TUS decreased somatosensory evoked potential amplitude [11], enhanced sensory discrimination task performance [11], and induced tactile sensations in contralateral hand 
regions $[12,13]$. Overall, these findings suggest that TUS can modulate sensorimotor functions in humans.

To date, whether TUS modifies the excitability of deeper brain structures in humans is unknown. Although one recent study showed somatosensory evoked potentials modulation by TUS on the thalamus [14], no study has assessed the possible effects of brainstem insonation. A reliable neurophysiological tool to investigate human brainstem circuits is the trigeminal blink reflex, elicited by electrical stimulation of the supraorbital nerve and mediated by large myelinated non-nociceptive fibers. The blink reflex is characterized by an early ipsilateral component (R1), which originates from an oligosynaptic pontine circuit, followed by a bilateral late component (R2), which reflects the activity of a polysynaptic circuit in the medullary reticular area [15-18]. Brainstem circuit excitability can be assessed through the $\mathrm{R} 2$ recovery cycle, a non-invasive neurophysiological technique based on paired stimulation at different interstimulus intervals (ISI) $[15,19,20]$. Various neural systems are involved in the control and modulation of the blink reflex, including descending pathways from cortical and subcortical areas [16,21-26].

In this study, we tested the effects of TUS on trigeminal blink reflex excitability, as assessed by the blink reflex recovery cycle, in healthy humans. For this purpose, we delivered TUS in different sessions targeting three brainstem areas, i.e., substantia nigra, superior colliculus, and nucleus raphe magnus. Due to the different roles of the three aforementioned nuclei on blink reflex circuit, e.g., the substantia nigra inhibits the superior colliculus which in turn excites the inhibitory activity of the nucleus raphe magnus [21,22], we hypothesize facilitatory or inhibitory effects of TUS on blink reflex excitability depending on the targeted area. A control session with sham stimulation was also performed. The blink reflex recovery cycle was assessed before and at two time-points after TUS, thus allowing us to investigate both the short- and long-term effects of TUS. A better understanding of the effects of TUS on blink reflex may guide novel non-invasive approaches for investigating and neuromodulating brainstem functions in healthy subjects and in pathological conditions [25].

\section{Materials and Methods}

\subsection{Participants}

Sixteen healthy subjects ( 7 females; mean age \pm 1 SD: $27 \pm 2.3$ years; age range: 23-33 years) were consecutively enrolled at the Department of Human Neurosciences, Sapienza University of Rome. All subjects were right-handed as assessed by the Edinburgh Handedness Inventory (score $\geq 60$ ) [27], and none presented a history of any neurological, psychiatric, eye, or eyelid disorder or were taking any drug potentially acting on the central nervous system. Other exclusion criteria were jet lag, irregular working hours, sleep restrictions in the last week or past drug abuse. All participants gave their informed consent to the experimental procedures, which were approved by the local ethics committee and conducted in accordance with the Declaration of Helsinki.

\subsection{Transcranial Ultrasound Stimulation}

TUS was performed with a 4 P1 probe (Siemens S2000 apparatus, Siemens Medical, Solutions, WA, USA) with standard diagnostic transcranial imaging settings. B-Mode transtemporal right-sided imaging was obtained in the standard axial planes (midbrain, upper and lower pons, and cella media planes) [28] to identify the structures of interest. TUS insonation was performed for $180 \mathrm{~s}$, with the mechanical index set to $100 \%$ (1.4; TIC $2.2)$, reducing the probe frequency to as low as possible $(1.75 \mathrm{MHz})$ to maximize ultrasound beam penetration. Pulsed-wave Doppler (2 MHz, PRF 4340) was also switched on during insonation allowing the visualization of B-Mode imaging in real time simultaneously with pulse-wave Doppler. For each stimulation session, the following structures were identified, kept on screen, and insonated: the superior colliculus, nucleus raphe magnus, and substantia nigra [28] (Figure 1). Neuroanatomical landmarks were used to focus 
ultrasounds according to previous publications on the topic [29]. The sham stimulation was performed with the "freezing" machine ultrasound option.

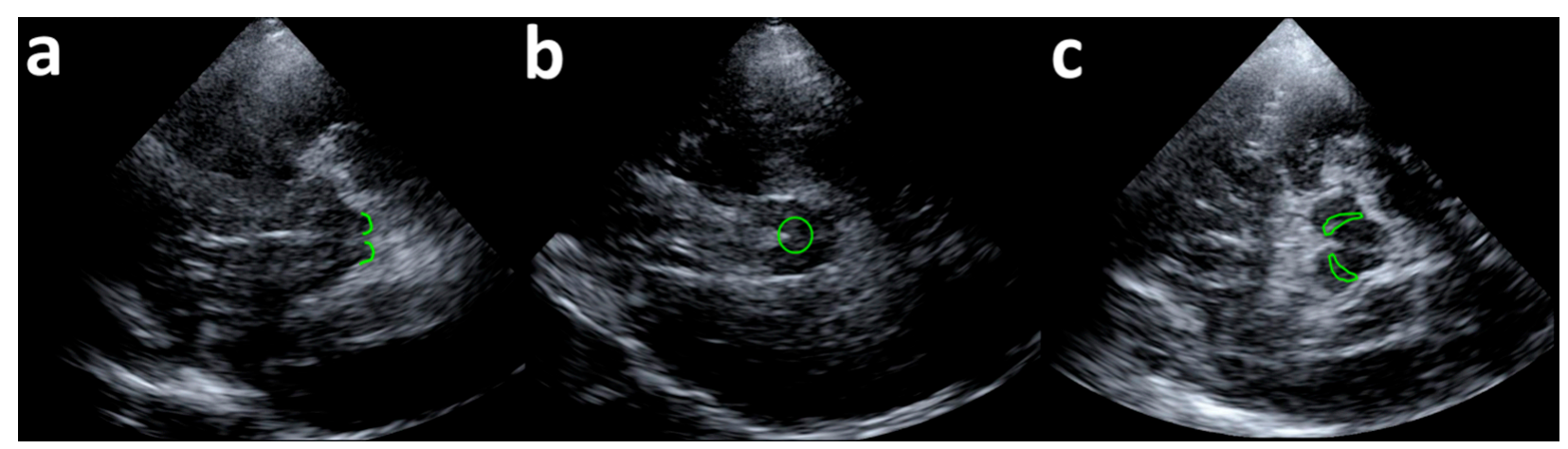

Figure 1. Ultrasound visualization of target brainstem nuclei. (a) Superior colliculus (SC); (b) Nucleus raphe magnus (NRM); (c) Substantia nigra (SN). The size of the targeted area was $\sim 15 \mathrm{~mm}$ and brainstem nuclei were centred on the screen.

An acoustic model was implemented to evaluate the attenuation of the ultrasound beam properties by the skull bone and to estimate the pressure value produced at the brainstem level. For the simulation, we used the characteristics of the transducer adopted in this study, a bone speed of sound of $3476 \mathrm{~m} / \mathrm{s}$ and a midbrain speed of sound of $1546.3 \mathrm{~m} / \mathrm{s}$. The modelling was computed using the K-Wave toolbox in MATLAB [30] and the estimated pressure of the ultrasound beam in the brainstem resulted $152 \mathrm{kPa}$ (Figure 2).

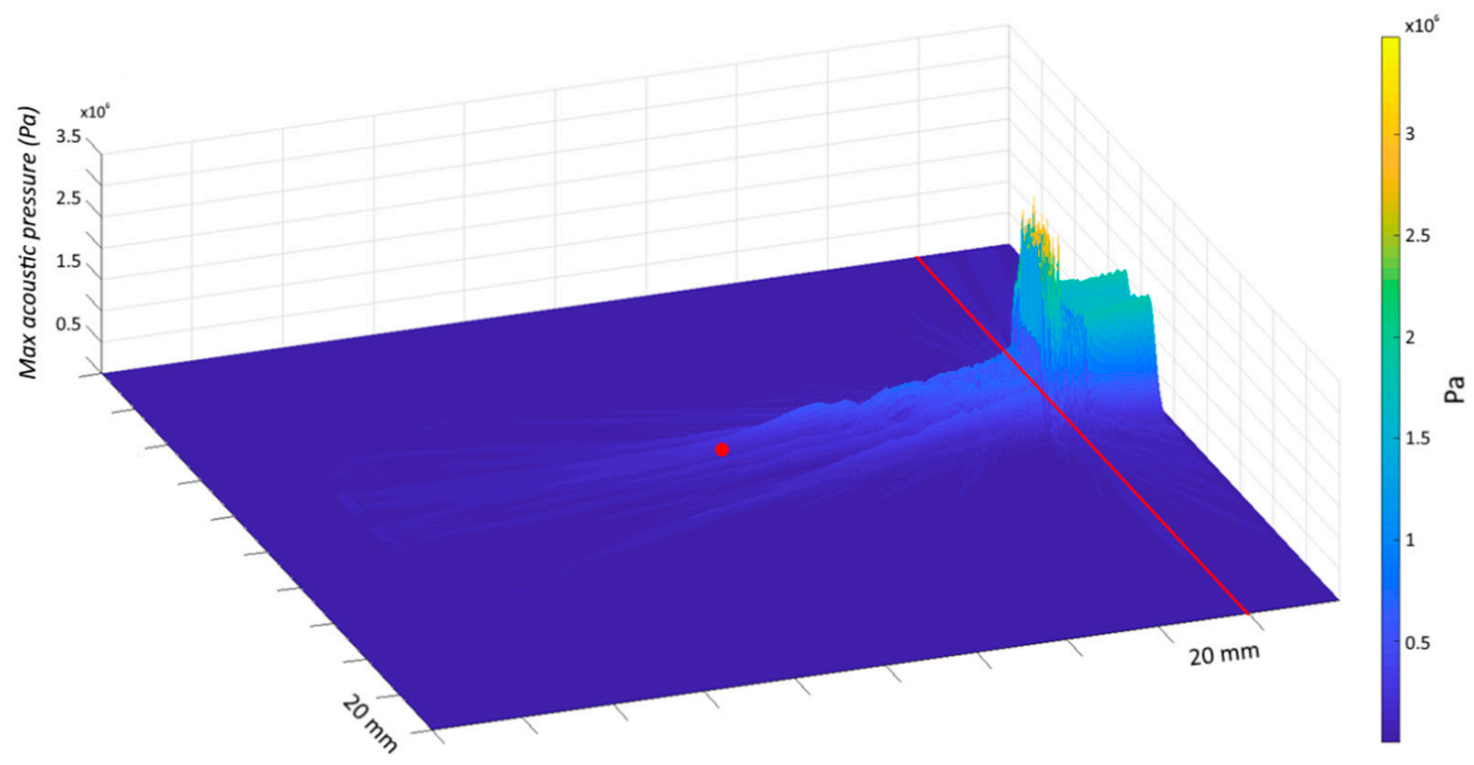

Figure 2. Ultrasound beam simulation. Pressure field of the ultrasound beam from the temporal bone (site of probe application—red line) to the target area in the brainstem (distance of $\sim 80 \mathrm{~mm}$-red dot). The coordinates of the target area have been determined from the brain scan atlas created by Neuromorphometrics, Inc. (https://scalablebrainatlas.incf. org/human/NMM1103, accessed on 5 February 2021), following the methods described in [31]. Based on our model, the pressure wave was reduced by $\sim 75 \%$ from the temporal bone to the targeted site due to the skull attenuation (temporal bone thickness: $5 \mathrm{~mm}$ ) and the estimated pressure of the ultrasound beam in the brainstem resulted $152 \mathrm{kPa}$.

\subsection{Blink Reflex and R2 Recovery Cycle}

EMG recordings were obtained from the orbicularis oculi muscle bilaterally using surface electrodes. The cathode was placed over the muscle, the anode at the lateral angle of the eye. EMG signals were amplified using D360 amplifiers (Digitimer, Welwyn, UK), bandpass filtered $(53-2500 \mathrm{~Hz})$, analog-to-digital converted using a $1401 \mathrm{AD}$ converter 
(CED, Cambridge, UK) at a sample rate of $5000 \mathrm{~Hz}$, and collected on a computer. Electrical stimulation was applied to the right supraorbital nerve with a bipolar stimulating electrode and constant current generator (Digitimer). The supraorbital nerve was stimulated immediately above the supraorbital notch with the cathode positioned above the supraorbital foramen and the anode about $2 \mathrm{~cm}$ higher and laterally rotated. All stimuli had a $0.2 \mathrm{~ms}$ duration and stimulus intensity was set at approximately 3 times the R2 threshold (lowest intensity with an $\mathrm{R} 2$ of $\geq 50 \mu \mathrm{V}$ response in at least 5 out of 10 trials). The blink reflex response to paired stimulation was assessed at ISI of 250 and $500 \mathrm{~ms}$ as this is a commonly applied methodology to test the inhibitory phase of the R2 recovery cycle [32-34]. Trials with background EMG $\geq 100 \mu \mathrm{V}$ were rejected online. Data were analyzed offline using Signal software (Cambridge Electronic Design, UK). Raw blink recordings were DC-corrected, rectified, and averaged [35]. The onset and offset of the conditioned and unconditioned R2 responses were manually determined for each trial. The area under the curve of the rectified EMG was then measured and the ratio between conditioned and unconditioned R2 areas was calculated (Figure 3). Measures were analyzed offline by two investigators who were unaware of the session type.
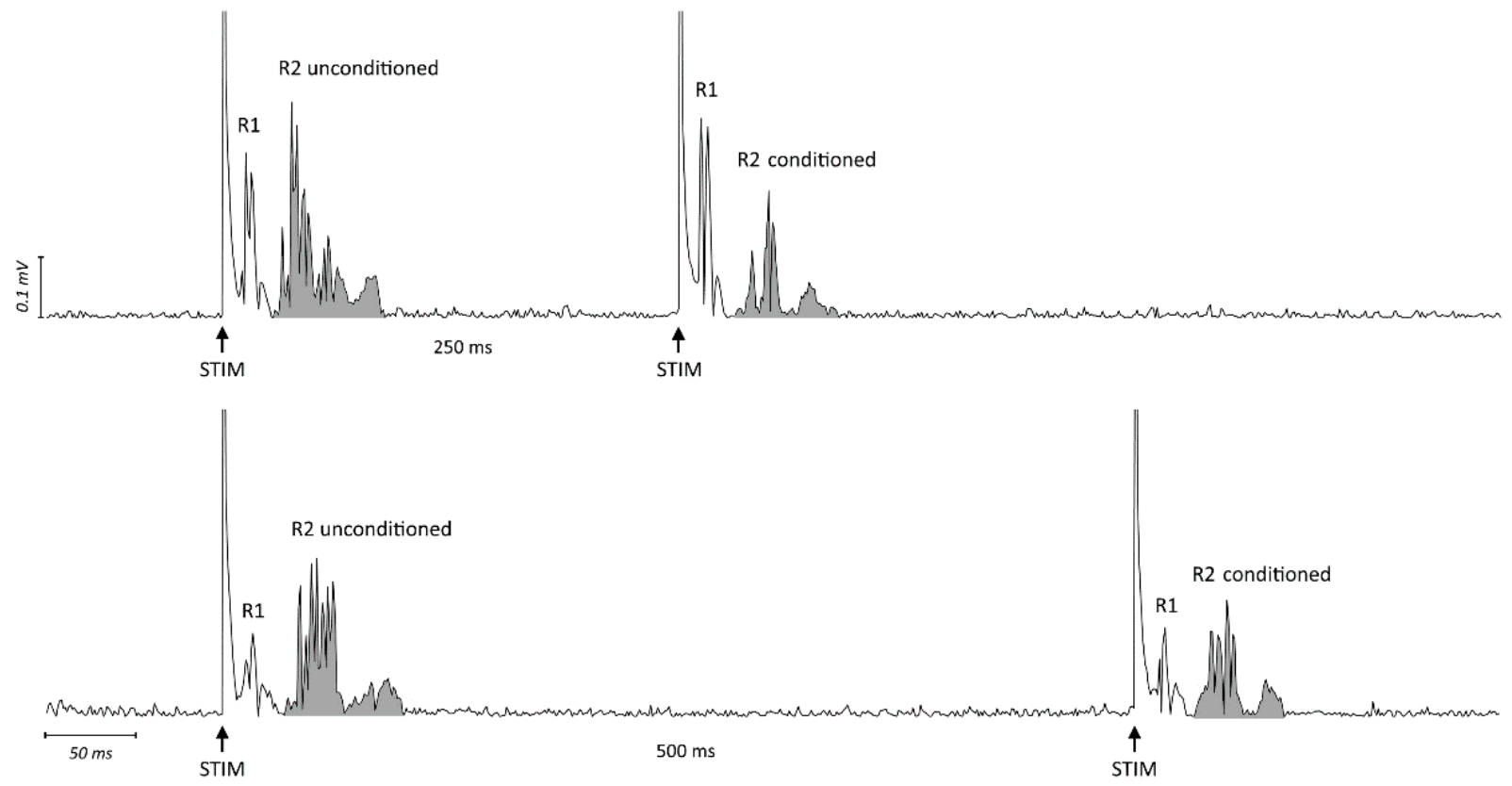

Figure 3. Raw EMG traces of blink reflex response to paired stimulation at ISI $250 \mathrm{~ms}$ (upper trace) and $500 \mathrm{~ms}$ (lower trace) in one representative subject. Shown are rectified traces recorded from the right orbicularis oculi muscle. The arrows indicate the timing of electric stimulation (STIM). The grey area represents the area under the curve (AUC) of the rectified EMG for the unconditioned and conditioned R2. The ratios between the AUC of conditioned R2 (paired stimulation) and unconditioned R2 (first stimulation) in the upper and lower trace were 0.44 and 0.67 , respectively.

\subsection{Experimental Procedure}

Participants were seated on a chair, at rest, with their eyes open in a quiet room with normal indoor lighting. All subjects underwent four different experimental sessions, conducted in a random order and on separate days: (1) superior colliculus-TUS; (2) nucleus raphe magnus-TUS; (3) substantia nigra-TUS; (4) sham-TUS. In each session, both participants and investigators were blind to the stimulation condition, except for the researcher who delivered TUS. The R2 recovery cycle was assessed by 10 paired stimuli ( 5 with $250 \mathrm{~ms}$ ISI and 5 with $500 \mathrm{~ms}$ ISI) applied in a random order, with a 30-45 sintertrial interval to avoid habituation. Blink reflex responses were recorded before (T0), 3 (T1—early effect), and $30 \mathrm{~min}$ (T2-late effect) after TUS. 


\subsection{Statistical Analysis}

Data were analyzed using repeated measures analyses of variance (rmANOVAs). A first rmANOVA with 'CONDITION' (4 levels: superior colliculus-TUS, nucleus raphe magnus-TUS, substantia nigra-TUS, and sham-TUS), 'SIDE' (2 levels: right and left) and 'TIME-POINT' ( 3 levels: T0, T1, and T2) was used to compare the area of unconditioned R2 between the different sessions and time-points. Another rmANOVA with 'CONDITION', 'SIDE', 'TIME-POINT', and 'ISI' (2 levels: $250 \mathrm{~ms}$ and $500 \mathrm{~ms}$ ) was applied to assess possible changes in the area of conditioned R2. Paired t-tests were also used to specifically compare the area of unconditioned R2 at T0 between sessions. Greenhouse-Geisser corrections were applied when a violation of sphericity in Mauchly's tests was detected. Possible neurophysiological correlations were assessed using Pearson's correlation test. The level of significance was set at $p<0.05$ and Tukey's test was used in post-hoc analysis to correct for multiple comparisons. Statistical analysis was performed using STATISTICA (TIBCO software inc., Palo Alto, CA, USA).

\section{Results}

The experimental procedures were well tolerated by all subjects and none reported adverse effects or any skin sensation during or after TUS. In all subjects, the electrical stimulation of the right supraorbital nerve evoked a reliable R2 response in the ipsilateral and contralateral orbicularis oculi muscle, while the intensity used was insufficient to induce a reliable R1 response.

As expected, the rmANOVA conducted on the unconditioned R2 only showed a significant effect of the factor 'SIDE' $\left(\mathrm{F}_{1,15}=19.81, p<0.001\right)$, indicating that the R2 size was higher in the muscle ipsilateral (right) than contralateral to the stimulation. The main factors 'CONDITION' $\left(\mathrm{F}_{3,45}=1.80, p=0.16\right)$ and 'TIME-POINT' $\left(\mathrm{F}_{2,30}=2.71, p=0.09\right)$, as well as the 'SIDE' $\times$ 'CONDITION' $\left(\mathrm{F}_{3,45}=0.56, p=0.64\right)$, 'SIDE' $\times$ 'TIME-POINT'

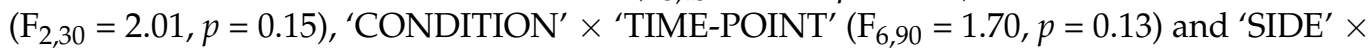
'CONDITION' $\times$ 'TIME-POINT' $\left(\mathrm{F}_{6,90}=1.69, p=0.13\right)$ interactions were non-significant.

The rmANOVA conducted on the conditioned R2 highlighted a significant 'CONDITION' $\times$ 'TIME-POINT' interaction $\left(\mathrm{F}_{6,90}=2.92, p=0.01\right)$, indicating a time-dependent modulation of the R2 component depending on the stimulation condition. Post-hoc analysis demonstrated a higher conditioned R2 area at T1 than at T0 $(p<0.01)$ in the superior colliculus-TUS session, while no difference was present between T0 and T2 $(p=0.41)$ (Figure 4; Figure S1 shows individual data for superior colliculus-TUS). In contrast, in all other stimulation conditions the conditioned R2 area did not change after TUS (nucleus raphe magnus-TUS: T0 vs. T1 $p=0.99$, T0 vs. T2 $p=1.0$; substantia nigra-TUS: T0 vs. T1 $p=0.99$, T0 vs. T2 $p=1.0$; sham-TUS: T0 vs. T1 $p=0.99$, T0 vs. T2 $p=0.99$ ). As expected, the analysis also demonstrated a significant effect of the main factors, 'SIDE' $\left(\mathrm{F}_{1,15}=11.92, p<0.01\right)$ and 'ISI' $\left(\mathrm{F}_{1,15}=42.51, p<0.001\right)$, suggesting that the area of the conditioned R2 component was lower in the muscle ipsilateral (right) than contralateral to the stimulation, and lower at $250 \mathrm{~ms}$ than $500 \mathrm{~ms}$ ISI in all stimulation conditions and time-points considered. Finally, the rmANOVA did not show any effect of 'CONDITION' or 'TIME-POINT' or other interactions between factors ( $p$ always $>0.05$, Table 1$)$. In summary, the results suggest an increased conditioned R2 area immediately after superior colliculus-TUS, reflecting brainstem circuit excitability changes. 


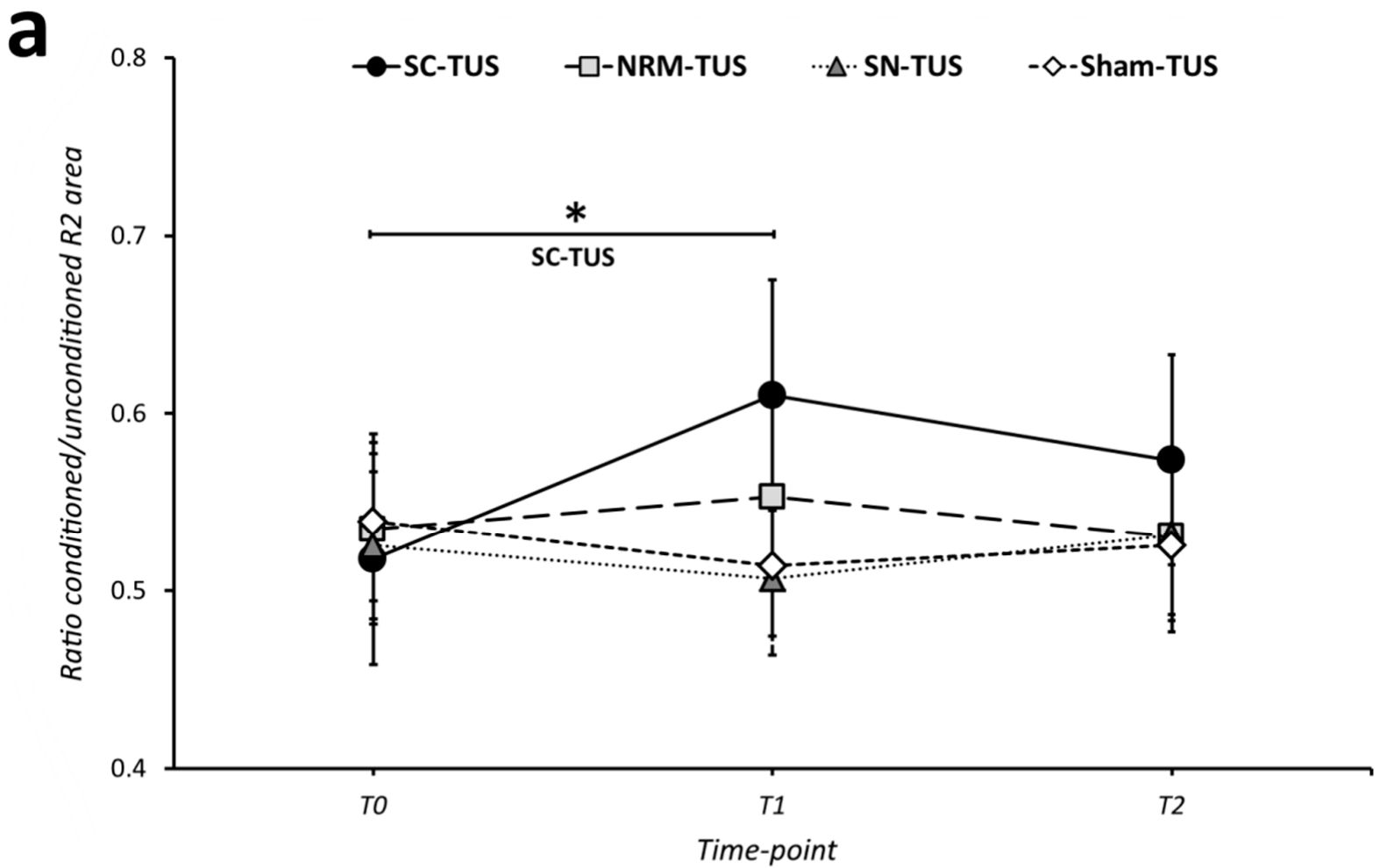

b

SN-TUS

Sham-TUS
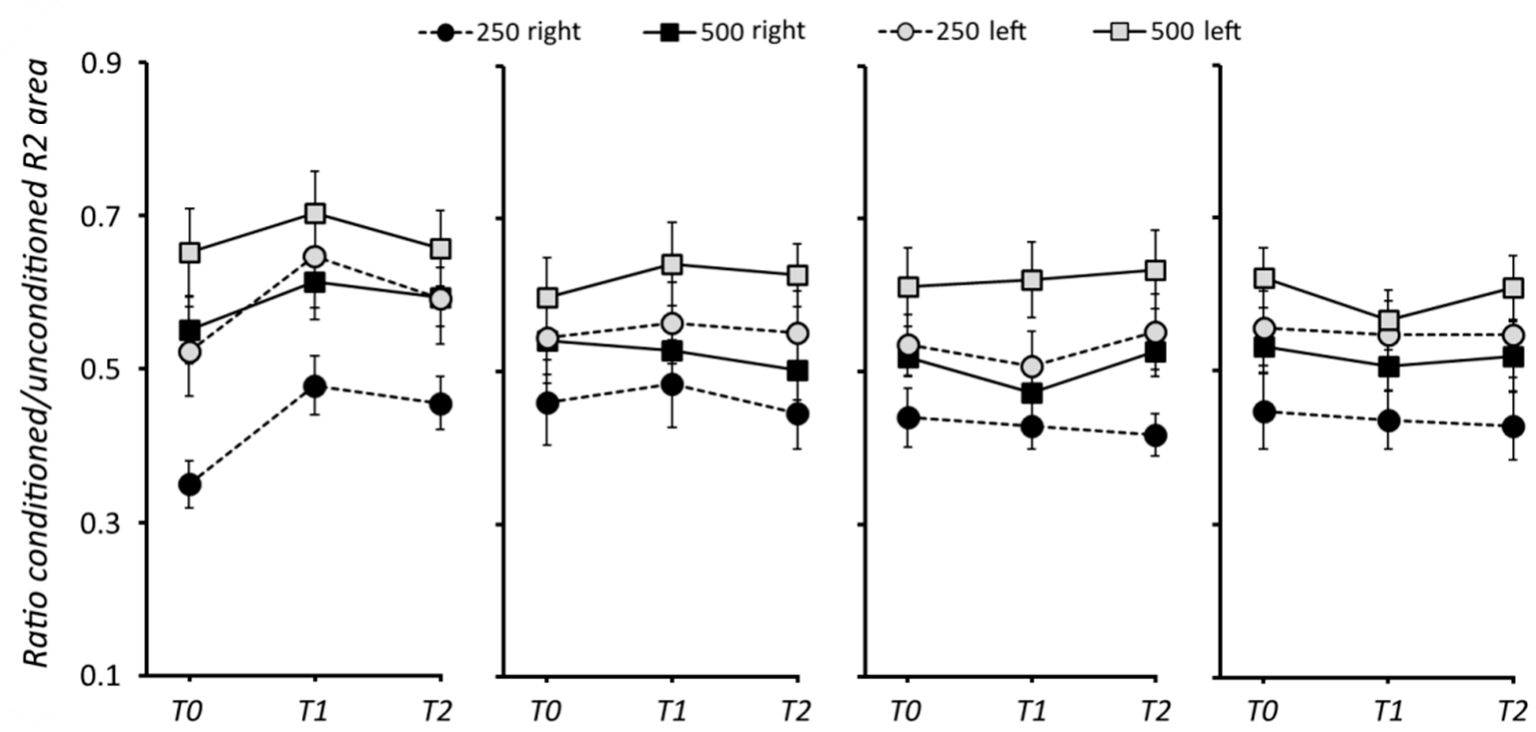

Figure 4. Effects of transcranial ultrasound stimulation on blink reflex excitability. (a) Transcranial ultrasound stimulation (TUS) delivered for $180 \mathrm{~s}$ on the superior colliculus (SC) increased the conditioned R2 area (i.e., lower R2 inhibition) at $3 \mathrm{~min}$ (T1) post-stimulation. In contrast, nucleus raphe magnus (NRM), substantia nigra (SN), and sham-TUS had no significant effects on blink reflex excitability. Since statistical analysis demonstrated that the effect was independent of the interstimulus interval (ISI) tested ( 250 or $500 \mathrm{~ms}$ ) and R2 recording side (right or left), the panel shows averaged values. Error bars reflect the standard error of the mean. The asterisk denotes significant differences at post-hoc analysis. (b) Effects of SC, NRM, SN, and sham-TUS on the conditioned R2 area at the two ISI tested in the right and left side. Error bars reflect the standard error of the mean. T0: before TUS; T1: 3 min after TUS; T2: 30 min after TUS. 
Table 1. Results of rmANOVA conducted on unconditioned R2 values.

\begin{tabular}{|c|c|c|c|}
\hline Factors and Interactions & $\mathbf{F}$ & $d, f$ & $p$ \\
\hline 'SIDE' & 11.92 & 1,15 & $<0.01$ \\
\hline 'CONDITION’ & 1.02 & 3,45 & 0.39 \\
\hline 'TIME-POINT' & 2.09 & 2,30 & 0.14 \\
\hline ‘ISI' & 42.51 & 1,15 & $<0.001$ \\
\hline 'SIDE' $\times$ 'CONDITION' & 0.13 & 3,45 & 0.94 \\
\hline 'SIDE' $\times$ 'TIME-POINT' & 0.16 & 2,30 & 0.86 \\
\hline 'CONDITION' $\times$ ‘TIME-POINT' & 2.92 & 6,90 & 0.01 \\
\hline 'SIDE' $\times$ 'ISI' & 2.40 & 1,15 & 0.14 \\
\hline 'CONDITION' $\times$ 'ISI' & 1.55 & 3,45 & 0.21 \\
\hline ‘TIME-POINT’ $\times$ 'ISI' & 1.03 & 2,30 & 0.37 \\
\hline 'SIDE' $\times$ 'CONDITION' $\times$ ‘TIME-POINT' & 0.70 & 6,90 & 0.65 \\
\hline 'SIDE’ $\times$ ‘CONDITION' $\times$ 'ISI' & 2.53 & 3,45 & 0.07 \\
\hline 'SIDE' $\times$ 'TIME-POINT' $\times$ 'ISI' & 1.16 & 2,30 & 0.33 \\
\hline 'CONDITION' $\times$ 'TIME-POINT' $\times$ 'ISI' & 0.69 & 6,90 & 0.66 \\
\hline 'SIDE' $\times$ 'CONDITION' $\times$ 'TIME-POINT' $\times$ 'ISI' & 1.33 & 6,90 & 0.25 \\
\hline
\end{tabular}

Finally, we tested whether the effects of superior colliculus-TUS (ratio conditioned R2 T1/T0, averaged across side and ISI) depended on the baseline level of blink reflex excitability in the same session (conditioned R2 at T0). The correlation analysis demonstrated no significant relationship between these measures $(r=-0.36, p=0.17)$.

\section{Discussion}

In the present study, we demonstrated that TUS targeting the superior colliculus, but not other brainstem areas, increased the conditioned $\mathrm{R} 2$ component area. This effect was present immediately after TUS, but not at a later time. Results were independent of the recording side (right or left) and the ISI tested ( 250 or $500 \mathrm{~ms})$. These data suggest that superior colliculus-TUS modulates the excitability of the trigeminal blink reflex circuit in humans.

The double-blind, crossover, randomized, and sham-controlled experimental design allowed us to exclude that our results were biased by confounding factors. First, we carefully followed international standard procedures and we used well-known neuroanatomical landmarks to identify and target the various brainstem nuclei before applying TUS [28,29]. Our acoustic model demonstrates that despite the pressure wave being attenuated by the temporal bone, the targeted brainstem areas still received a proportion of the original ultrasound beam. Both participants and researchers who collected and analyzed the neurophysiological data were blinded to the stimulation conditions. The four experimental sessions were conducted at least $24 \mathrm{~h}$ apart, thus excluding possible carryover effects of TUS. In addition, sham-TUS did not induce any relevant effect on blink reflex recovery cycle, thus excluding the contribution of unspecific attentive or cognitive factors to blink reflex recovery cycle changes. In the latter regard, the area of unconditioned R2 was similar between the different sessions and time-points, possibly indicating no influence of unspecific arousal changes [36,37]. Finally, brainstem excitability at baseline was comparable between the different stimulation conditions, as shown by the similar unconditioned and conditioned R2 values before TUS in the various experimental sessions. We conclude that our results are essentially due to the putative physiological effects of TUS on the excitability of brainstem circuits controlling the blink reflex.

The main result of the study was the increased conditioned R2 area (both at 250 and $500 \mathrm{~ms}$ ) immediately after TUS delivered on the superior colliculus, thus indicating early and short-lasting brainstem excitability changes. According to previous experimental studies on animals, the superior colliculus is thought to have a prominent role in modulating brainstem excitability $[21,22,38]$. The superior colliculus is a small, bilateral, and symmetrical nucleus in the tegmental midbrain area composed of several neuronal layers with intermediate and deep layers [22,39-41]. It receives inhibitory input via GABA projections 
from the substantia nigra and provides an excitatory output to the nucleus raphe magnus, which in turn inhibits brainstem excitability through 5 HT neurotransmission [21,22]. Accordingly, both direct (by stimulation of intermediate-to-deeper layers) and indirect (through inhibition of the substantia nigra) excitation of the superior colliculus caused a reduction in brainstem excitability in animals [21,22,39].

Several hypotheses may explain the increased conditioned R2 area after TUS delivered on the superior colliculus. The first hypothesis is that TUS exerts a direct inhibitory effect on deeper layers and output fibres of the superior colliculus projecting to the nucleus raphe magnus. Accordingly, nucleus raphe magnus activity would be reduced, resulting in a less inhibited blink reflex (Figure 5). However, evidence from animal studies indicates that ultrasound-induced currents determine excitatory rather than inhibitory effects, as demonstrated by increased neuronal firing $[1-3,42,43]$ and enhanced neurotrophic factor levels $[2,44,45]$. Similar evidence in humans suggests that TUS has excitatory effects when it is delivered with the parameters we used (including frequency and stimulation duration) $[6,7,10]$. Hence, the hypothesis that brainstem excitability changes after TUS delivered on the superior colliculus are due to inhibitory effects on the deeper layers and output fibres of this nucleus is unlikely. A second hypothesis is that the increased R2 area after superior colliculus-TUS is due to excitatory effects of the stimulation on GABAergic inhibitory synapses between substantia nigra and superior colliculus neurons (Figure 4) [21,22]. In this case, TUS would indirectly inhibit superior colliculus output, thus producing nucleus raphe magnus activity depression and reduced inhibition of the blink reflex. However, we found no significant change in blink reflex excitability after substantia nigra-TUS. Therefore, we also exclude the hypothesis that TUS produces its effects through excitation of inhibitory projections from the substantia nigra to the superior colliculus. The hypothesis we favour is that TUS exerts excitatory activity on inhibitory interneurons within the superior colliculus. There is evidence that GABAergic interneurons within the superior colliculus downregulate the activity of the nucleus itself [40,46-49]. Also, a recent study demonstrated that TUS increases GABAergic inhibition at the cortical level [9]. Accordingly, TUS may enhance the activity of superior colliculus GABAergic interneurons and, in turn, produce an overall reduction in superior colliculus output to the nucleus raphe magnus, thus leading to an increased R2 area (Figure 4). We cannot exclude, however, that other inhibitory interneurons in the superior colliculus may also contribute to TUS effects. Since superior colliculus-TUS effects were only present early after stimulation and did not persist later in time, we speculate that TUS only produces short-lasting modifications in the excitability of these neurons rather than stable long-term synaptic plasticity mechanisms in blink reflex circuits [35,50]. These data are overall in line with the results of animal studies indicating transient effects of experimental manipulations on brainstem function, lasting from milliseconds up to two hours according to the methodology adopted [21,22,39].

The lack of substantia nigra and nucleus raphe magnus-TUS effects deserves a separate comment. Our results showed that the conditioned R2 area did not change before or after substantia nigra and nucleus raphe magnus stimulation, indicating comparable brainstem excitability before and after stimulation. We exclude that the lack of effect was due to a possible weak role of substantia nigra and nucleus raphe magnus in influencing the trigeminal blink reflex since several studies have demonstrated that they are both key structures in brainstem circuits [21,22]. Because of the relatively low focality of TUS, it is possible that substantia nigra or nucleus raphe magnus insonation had variable effects due to the coactivation of their facilitatory and inhibitory connections with multiple brain structures, resulting in no significant change in the trigeminal blink reflex excitability. Considering the results provided by our modelling, however, we favor the hypothesis that different brainstem areas have a variable sensitivity to the mechanical energy of the ultrasound beam. While relatively low pressure suffices to modulate superior colliculus interneurons, a higher energy is possibly needed to modify the activity of substantia nigra and nucleus raphe magnus. Specific biomechanical and bioelectrical properties of superior colliculus interneurons would make them particularly sensitive to TUS. 


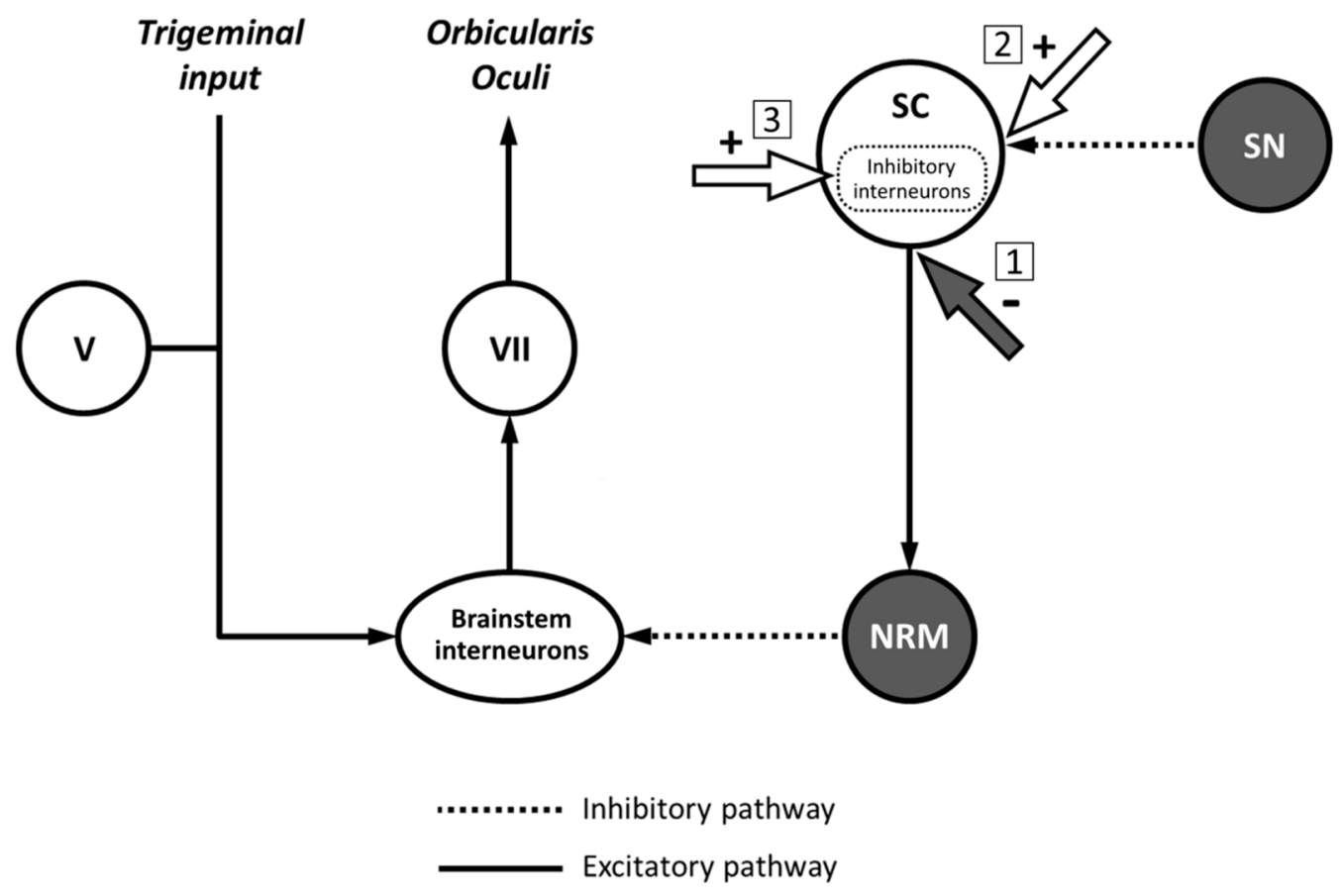

Figure 5. Possible mechanisms of action of transcranial ultrasound stimulation on the blink reflex circuit. Schematic representation of nuclei and circuits controlling blink reflex excitability (adapted from [21]) and possible mechanisms to explain the finding of reduced blink reflex inhibition after transcranial ultrasound stimulation (TUS). When delivered on the superior colliculus (SC), TUS may directly inhibit fibers originating from the SC and projecting to the nucleus raphe magnus (NRM) (1). Another possibility is that TUS exerts an excitatory effect at the level of GABAergic inhibitory synapses between substantia nigra (SN) projections and SC neurons (2). A third possible mechanism implies the facilitation of the activity of inhibitory interneurons located within the SC, thus reducing the output of the nucleus itself (3).

This study has some limitations. First, TUS has a relatively low spatial resolution [6,51], and thus we cannot exclude that regions outside of the targeted nuclei at brainstem level, where various structures and projections are tightly packed, were also insonated. Moreover, we have not performed anatomical or functional imaging investigations to better target the nuclei of interest [28]. Also, a recent study in animals found that ultrasonic neuromodulation possibly modifies cortical activity through indirect auditory mechanisms [52]. However, we believe this is unlikely to explain our results since the transducer position was similar in the different stimulation conditions, but the R2 changes occurred only during the superior colliculus-TUS. Finally, the superior colliculus is known to modulate not only eyelid blink but also saccadic eye movements [38]. However, monitoring eye movements was beyond the purpose of our study. Importantly, there is currently no standardized TUS protocol in humans, and different stimulation parameters (e.g., ultrasound power, duration, frequency, and stimulation pattern) may have variable results [6,7]. Therefore, our results are limited to the TUS parameters we used. In this regard, we cannot exclude that increasing the stimulation duration over $180 \mathrm{~s}$ determines a stronger superior colliculus modulation or effective changes in substantia nigra and nucleus raphe magnus activity. Future research may focus on these specific issues.

\section{Conclusions}

This is the first study investigating the effects of TUS on brainstem circuits in humans. We demonstrated that the conditioned R2 area increases early after the application of TUS on the superior colliculus suggesting a lack of brainstem excitability inhibition. The facilitatory effect of TUS on brainstem excitability may be explained by an excitatory action on GABAergic interneurons within the superior colliculus, while alternative hypotheses seem less likely. Overall, TUS is a safe, easy-to-use, and well-tolerated technique. A recent systematic review highlighted both excitatory and suppressive modulatory effects 
of TUS depending on the stimulation parameters and targeted areas [7]. Future studies assessing TUS effects on different deep and cortical neuronal circuits may provide further details on its mechanism of action. Again, the behavioral counterpart of brainstem TUS, e.g., changes in eye movements particularly due to the stimulation of superior colliculus, deserve further investigations [53]. Finally, TUS may be applied in pathological conditions characterized by altered brainstem function [25] to verify whether modulating abnormal brainstem excitability improves specific symptoms.

Supplementary Materials: The following are available online at https://www.mdpi.com/article/10 .3390/brainsci11050645/s1, Figure S1: Effects of superior colliculus-transcranial ultrasound stimulation (SC-TUS) on blink reflex excitability at the individual subject level.

Author Contributions: Conceptualization, M.B., A.G.; methodology, E.V., A.B.; formal analysis, A.G., E.C., D.C.; investigation, E.C., D.C., A.C., A.G.; data curation, G.P., B.C., S.P.; writing —original draft preparation, A.G.; writing—review and editing, M.B., A.B., E.V., G.D.S., B.C. All authors have read and agreed to the published version of the manuscript.

Funding: This research received no external funding.

Institutional Review Board Statement: The study was conducted according to the guidelines of the Declaration of Helsinki and approved by the local ethics committee.

Informed Consent Statement: Informed consent was obtained from all subjects involved in the study.

Data Availability Statement: The datasets analyzed during the current study are available from the corresponding author upon reasonable request.

Acknowledgments: The authors wish to thank all volunteers for their participation in this study.

Conflicts of Interest: The authors declare no conflict of interest.

\section{References}

1. Tyler, W.J.; Tufail, Y.; Finsterwald, M.; Tauchmann, M.L.; Olson, E.J.; Majestic, C. Remote Excitation of Neuronal Circuits Using Low-Intensity, Low-Frequency Ultrasound. PLoS ONE 2008, 3, e3511. [CrossRef] [PubMed]

2. Tufail, Y.; Matyushov, A.; Baldwin, N.; Tauchmann, M.L.; Georges, J.; Yoshihiro, A.; Tillery, S.I.H.; Tyler, W.J. Transcranial Pulsed Ultrasound Stimulates Intact Brain Circuits. Neuron 2010, 66, 681-694. [CrossRef]

3. Blackmore, J.; Shrivastava, S.; Sallet, J.; Butler, C.R.; Cleveland, R.O. Ultrasound Neuromodulation: A Review of Results, Mechanisms and Safety. Ultrasound Med. Biol. 2019, 45, 1509-1536. [CrossRef] [PubMed]

4. Kubanek, J.; Shukla, P.; Das, A.; Baccus, S.A.; Goodman, M.B. Ultrasound Elicits Behavioral Responses through Mechanical Effects on Neurons and Ion Channels in a Simple Nervous System. J. Neurosci. 2018, 38, 3081-3091. [CrossRef]

5. Krasovitski, B.; Frenkel, V.; Shoham, S.; Kimmel, E. Intramembrane Cavitation as a Unifying Mechanism for Ultrasound-Induced Bioeffects. Proc. Natl. Acad. Sci. USA 2011, 108, 3258-3263. [CrossRef]

6. Di Biase, L.; Falato, E.; Di Lazzaro, V. Transcranial Focused Ultrasound (TFUS) and Transcranial Unfocused Ultrasound (TUS) Neuromodulation: From Theoretical Principles to Stimulation Practices. Front. Neurol. 2019, 10, 549. [CrossRef]

7. Wang, P.; Zhang, J.; Yu, J.; Smith, C.; Feng, W. Brain Modulatory Effects by Low-Intensity Transcranial Ultrasound Stimulation (TUS): A Systematic Review on Both Animal and Human Studies. Front. Neurosci. 2019, 13, 696. [CrossRef]

8. Legon, W.; Bansal, P.; Tyshynsky, R.; Ai, L.; Mueller, J.K. Transcranial Focused Ultrasound Neuromodulation of the Human Primary Motor Cortex. Sci. Rep. 2018, 8, 10007. [CrossRef]

9. Fomenko, A.; Chen, K.-H.S.; Nankoo, J.-F.; Saravanamuttu, J.; Wang, Y.; El-Baba, M.; Xia, X.; Seerala, S.S.; Hynynen, K.; Lozano, A.M.; et al. Systematic Examination of Low-Intensity Ultrasound Parameters on Human Motor Cortex Excitability and Behavior. Elife 2020, 9. [CrossRef]

10. Gibson, B.C.; Sanguinetti, J.L.; Badran, B.W.; Yu, A.B.; Klein, E.P.; Abbott, C.C.; Hansberger, J.T.; Clark, V.P. Increased Excitability Induced in the Primary Motor Cortex by Transcranial Ultrasound Stimulation. Front. Neurol. 2018, 9, 1007. [CrossRef] [PubMed]

11. Legon, W.; Sato, T.F.; Opitz, A.; Mueller, J.; Barbour, A.; Williams, A.; Tyler, W.J. Transcranial Focused Ultrasound Modulates the Activity of Primary Somatosensory Cortex in Humans. Nat. Neurosci. 2014, 17, 322-329. [CrossRef]

12. Lee, W.; Kim, H.; Jung, Y.; Song, I.-U.; Chung, Y.A.; Yoo, S.-S. Image-Guided Transcranial Focused Ultrasound Stimulates Human Primary Somatosensory Cortex. Sci. Rep. 2015, 5, 8743. [CrossRef]

13. Lee, W.; Chung, Y.A.; Jung, Y.; Song, I.-U.; Yoo, S.-S. Simultaneous Acoustic Stimulation of Human Primary and Secondary Somatosensory Cortices Using Transcranial Focused Ultrasound. BMC Neurosci. 2016, 17, 68. [CrossRef] [PubMed]

14. Legon, W.; Ai, L.; Bansal, P.; Mueller, J.K. Neuromodulation with Single-Element Transcranial Focused Ultrasound in Human Thalamus. Hum. Brain Mapp. 2018, 39, 1995-2006. [CrossRef] 
15. Berardelli, A.; Cruccu, G.; Manfredi, M.; Rothwell, J.C.; Day, B.L.; Marsden, C.D. The Corneal Reflex and the R2 Component of the Blink Reflex. Neurology 1985, 35, 797-801. [CrossRef] [PubMed]

16. Cruccu, G.; Iannetti, G.D.; Marx, J.J.; Thoemke, F.; Truini, A.; Fitzek, S.; Galeotti, F.; Urban, P.P.; Romaniello, A.; Stoeter, P.; et al. Brainstem Reflex Circuits Revisited. Brain 2005, 128, 386-394. [CrossRef]

17. Valls-Solé, J. Neurophysiological Assessment of Trigeminal Nerve Reflexes in Disorders of Central and Peripheral Nervous System. Clin. Neurophysiol. 2005, 116, 2255-2265. [CrossRef] [PubMed]

18. Cruccu, G.; Deuschl, G. The Clinical Use of Brainstem Reflexes and Hand-Muscle Reflexes. Clin. Neurophysiol. 2000, 111, 371-387. [CrossRef]

19. Boelhouwer, A.J. Blink Reflexes and Preparation. Biol. Psychol. 1982, 14, 277-285. [CrossRef]

20. Valls-Solé, J.; Valldeoriola, F.; Tolosa, E.; Marti, M.J. Distinctive Abnormalities of Facial Reflexes in Patients with Progressive Supranuclear Palsy. Brain 1997, 120 Pt 10, 1877-1883. [CrossRef]

21. Basso, M.A.; Evinger, C. An Explanation for Reflex Blink Hyperexcitability in Parkinson's Disease. II. Nucleus Raphe Magnus. J. Neurosci. 1996, 16, 7318-7330. [CrossRef]

22. Basso, M.A.; Powers, A.S.; Evinger, C. An Explanation for Reflex Blink Hyperexcitability in Parkinson's Disease. I. Superior Colliculus. J. Neurosci. 1996, 16, 7308-7317. [CrossRef]

23. Valls-Sole, J. Assessment of Excitability in Brainstem Circuits Mediating the Blink Reflex and the Startle Reaction. Clin. Neurophysiol. 2012, 123, 13-20. [CrossRef]

24. Bologna, M.; Fabbrini, G.; Marsili, L.; Defazio, G.; Thompson, P.D.; Berardelli, A. Facial Bradykinesia. J. Neurol. Neurosurg. Psychiatry 2013, 84, 681-685. [CrossRef]

25. Bologna, M.; Berardelli, A. Brainstem Avenues in Parkinson's Disease Research. Clin. Neurophysiol. 2019, 130, 554-555. [CrossRef]

26. Vo, L.; Drummond, P.D. Coexistence of Ipsilateral Pain-Inhibitory and Facilitatory Processes after High-Frequency Electrical Stimulation. Eur. J. Pain 2014, 18, 376-385. [CrossRef]

27. Oldfield, R.C. The Assessment and Analysis of Handedness: The Edinburgh Inventory. Neuropsychologia 1971, 9, 97-113. [CrossRef]

28. Kern, R.; Perren, F.; Kreisel, S.; Szabo, K.; Hennerici, M.; Meairs, S. Multiplanar Transcranial Ultrasound Imaging: Standards, Landmarks and Correlation with Magnetic Resonance Imaging. Ultrasound Med. Biol. 2005, 31, 311-315. [CrossRef]

29. Becker, G.; Berg, D. Neuroimaging in Basal Ganglia Disorders: Perspectives for Transcranial Ultrasound. Mov. Disord. 2001, 16, 23-32. [CrossRef]

30. Treeby, B.E.; Cox, B.T. K-Wave: MATLAB Toolbox for the Simulation and Reconstruction of Photoacoustic Wave Fields. J. Biomed. Opt. 2010, 15, 021314. [CrossRef]

31. Strudwick Caviness, V.; Theodore Lange, N.; Makris, N.; Reed Herbert, M.; Nelson Kennedy, D. MRI-Based Brain Volumetrics: Emergence of a Developmental Brain Science. Brain Dev. 1999, 21, 289-295. [CrossRef]

32. Fong, P.-Y.; Edwards, M.J.; Lu, C.-S.; Chen, R.-S.; Rothwell, J.C.; Bhatia, K.P.; Huang, Y.-Z. Abnormal Blink Reflex Recovery Cycle in Manifesting and Nonmanifesting Carriers of the DYT1 Gene Mutation. Neuroreport 2016, 27, 1046-1049. [CrossRef]

33. Conte, A.; Fabbrini, G.; Belvisi, D.; Marsili, L.; Di Stasio, F.; Berardelli, A. Electrical Activation of the Orbicularis Oculi Muscle Does Not Increase the Effectiveness of Botulinum Toxin Type A in Patients with Blepharospasm. Eur. J. Neurol. 2010, 17, 449-455. [CrossRef]

34. Paparella, G.; Di Stefano, G.; Fasolino, A.; Di Pietro, G.; Colella, D.; Truini, A.; Cruccu, G.; Berardelli, A.; Bologna, M. Painful Stimulation Increases Spontaneous Blink Rate in Healthy Subjects. Sci. Rep. 2020, 10, 20014. [CrossRef]

35. Bologna, M.; Agostino, R.; Gregori, B.; Belvisi, D.; Manfredi, M.; Berardelli, A. Metaplasticity of the Human Trigeminal Blink Reflex. Eur. J. Neurosci. 2010, 32, 1707-1714. [CrossRef]

36. Kimura, J.; Harada, O. Excitability of the Orbicularis Oculi Reflex in All Night Sleep: Its Suppression in Non-Rapid Eye Movement and Recovery in Rapid Eye Movement Sleep. Electroencephalogr. Clin. Neurophysiol. 1972, 33, 369-377. [CrossRef]

37. Boelhouwer, A.J.; Brunia, C.H. Blink Reflexes and the State of Arousal. J. Neurol. Neurosurg. Psychiatry 1977, 40, 58-63. [CrossRef]

38. Goossens, H.H.; Van Opstal, A.J. Blink-Perturbed Saccades in Monkey. II. Superior Colliculus Activity. J. Neurophysiol. 2000, 83, 3430-3452. [CrossRef]

39. Gnadt, J.W.; Lu, S.M.; Breznen, B.; Basso, M.A.; Henriquez, V.M.; Evinger, C. Influence of the Superior Colliculus on the Primate Blink Reflex. Exp. Brain Res. 1997, 116, 389-398. [CrossRef]

40. Tardif, E.; Delacuisine, B.; Probst, A.; Clarke, S. Intrinsic Connectivity of Human Superior Colliculus. Exp. Brain Res. 2005, 166, 316-324. [CrossRef]

41. May, P.J. The Mammalian Superior Colliculus: Laminar Structure and Connections. Prog. Brain Res. 2006, 151, 321-378. [CrossRef]

42. Khraiche, M.L.; Phillips, W.B.; Jackson, N.; Muthuswamy, J. Ultrasound Induced Increase in Excitability of Single Neurons. In Proceedings of the Annual International Conference IEEE Engineering in Medicine and Biology Society, Vancouver, BC, Canada, 21-24 August 2008; pp. 4246-4249.

43. Kim, H.-B.; Swanberg, K.M.; Han, H.-S.; Kim, J.-C.; Kim, J.-W.; Lee, S.; Lee, C.J.; Maeng, S.; Kim, T.-S.; Park, J.-H. Prolonged Stimulation with Low-Intensity Ultrasound Induces Delayed Increases in Spontaneous Hippocampal Culture Spiking Activity. J. Neurosci. Res. 2017, 95, 885-896. [CrossRef]

44. Lin, W.-T.; Chen, R.-C.; Lu, W.-W.; Liu, S.-H.; Yang, F.-Y. Protective Effects of Low-Intensity Pulsed Ultrasound on AluminumInduced Cerebral Damage in Alzheimer's Disease Rat Model. Sci. Rep. 2015, 5, 9671. [CrossRef] 
45. Yang, F.-Y.; Lu, W.-W.; Lin, W.-T.; Chang, C.-W.; Huang, S.-L. Enhancement of Neurotrophic Factors in Astrocyte for Neuroprotective Effects in Brain Disorders Using Low-Intensity Pulsed Ultrasound Stimulation. Brain Stimul. 2015, 8, 465-473. [CrossRef]

46. Behan, M.; Kime, N.M. Intrinsic Circuitry in the Deep Layers of the Cat Superior Colliculus. Vis. Neurosci. 1996, 13, 1031-1042. [CrossRef]

47. Kaneda, K.; Isa, K.; Yanagawa, Y.; Isa, T. Nigral Inhibition of GABAergic Neurons in Mouse Superior Colliculus. J. Neurosci. 2008, 28, 11071-11078. [CrossRef]

48. Phongphanphanee, P.; Mizuno, F.; Lee, P.H.; Yanagawa, Y.; Isa, T.; Hall, W.C. A Circuit Model for Saccadic Suppression in the Superior Colliculus. J. Neurosci. 2011, 31, 1949-1954. [CrossRef]

49. Villalobos, C.A.; Wu, Q.; Lee, P.H.; May, P.J.; Basso, M.A. Parvalbumin and GABA Microcircuits in the Mouse Superior Colliculus. Front. Neural Circuits 2018, 12, 35. [CrossRef]

50. Mao, J.B.; Evinger, C. Long-Term Potentiation of the Human Blink Reflex. J. Neurosci. 2001, 21, RC151. [CrossRef]

51. Kubanek, J. Neuromodulation with Transcranial Focused Ultrasound. Neurosurg. Focus 2018, 44, E14. [CrossRef]

52. Sato, T.; Shapiro, M.G.; Tsao, D.Y. Ultrasonic Neuromodulation Causes Widespread Cortical Activation via an Indirect Auditory Mechanism. Neuron 2018, 98, 1031-1041.e2. [CrossRef] [PubMed]

53. Schiller, P.H.; True, S.D.; Conway, J.L. Effects of Frontal Eye Field and Superior Colliculus Ablations on Eye Movements. Science 1979, 206, 590-592. [CrossRef] [PubMed] 